\title{
Learning of Grasp Adaptation through Experience and Tactile Sensing
}

\author{
Miao $\mathrm{Li}^{1}$, Yasemin Bekiroglu${ }^{2}$, Danica Kragic ${ }^{2}$ and Aude Billard ${ }^{1}$
}

\begin{abstract}
To perform robust grasping, a multi-fingered robotic hand should be able to adapt its grasping configuration, i.e., how the object is grasped, to maintain the stability of the grasp. Such a change of grasp configuration is called grasp adaptation and it depends on the controller, the employed sensory feedback and the type of uncertainties inherit to the problem. This paper proposes a grasp adaptation strategy to deal with uncertainties about physical properties of objects, such as the object weight and the friction at the contact points. Based on an object-level impedance controller, a grasp stability estimator is first learned in the object frame. Once a grasp is predicted to be unstable by the stability estimator, a grasp adaptation strategy is triggered according to the similarity between the new grasp and the training examples. Experimental results demonstrate that our method improves the grasping performance on novel objects with different physical properties from those used for training.
\end{abstract}

\section{INTRODUCTION}

Robust grasping is one of the most important capabilities that robots are expected to have. This ability becomes particularly crucial when robots start to work in unstructured environments. Due to the discrepancy between the model and the actual world, or even the errors from the adequate robot control, the planned grasps will hardly be executed without any error. Thus the stability of the final grasp can not be guaranteed even though it has been taken into account in the grasp planing stage. Several works have attempted to integrate different sources of uncertainties in the grasp planing algorithms [1], [2]. But these analytical methods still can not ensure the stability of the final grasps during implementation.

In this paper, we follow another common approach to deal with grasping uncertainty by using sensory feedback in the grasp execution stage. To be more specific, we use the tactile sensing on the fingertips to provide information about the uncertainty of object mass, coefficient of friction, etc. Depending on the tactile sensing, the grasp adaptation mechanism is triggered to increase the probability of achieving a stable grasp. Comparing with other types of sensors, tactile sensors can be more informative to determine information on the physical properties of the grasped object, such as its mass, center of mass, distribution of mass and friction of coefficient. To build estimates of these quantities requires to develop exploratory tactile and manipulation strategies. This

\footnotetext{
${ }^{1} \mathrm{M}$. Li and A. Billard are with Learning Algorithms and Systems Laboratory (LASA) at École Polytechnique Fédérale de Lausanne (EPFL), Switzeland $\{$ miao.li, aude.billard\}eepfl.ch

${ }^{2}$ Y. Bekiroglu and D. Kragic are with KTH Royal Institute of Technology, Stockholm, Sweden, as members of the Computer Vision \& Active Perception Lab., Centre for Autonomous Systems \{yaseminb, danik\}@kth.se
}

paper proposes a method to adapt the grasp to deal with grasping uncertainties from these physical properties.

To cope with uncertainty in grasping, most previous works using sensory feedback focused on uncertainties originating from imprecise model of object's geometry [3], [4], object position and orientation [5], [6], [7], [8]. These geometric uncertainties can directly influence the relative configuration between the robotic hand and the object, upon which the grasp stability is built [9]. In these studies, either the visual feedback or the tactile (force) feedback is used to compensate for the uncertainties by locally adjusting the hand pose or the finger joint angles. The criteria for the adjustment is usually predefined by humans [8] or learned from a large grasp database in simulation [10], [11].

However, as studied in [2], [12], [13], the object physical properties that are usually unknown prior to contact, such as coefficient of friction, object weight and center of mass, can also significantly affect the stability or feasibility of the final grasps. For instance, a robotic hand will likely drop a grasped object when lifting it if the object is heavier than assumed, even though the hand is in the desired configuration. To alleviate the uncertainty of these physical properties of the object, an additional grasping force controller is usually employed to carefully adapt the grasping forces to unknown object weight and friction conditions at the contact points [14]. However, before transiting to force controller, a position controller is still required to correct the geometric uncertainties. The transition between position controller and force controller can result in various different discrete contact states, each of which usually needs to be treated separately [14], [15]. Moreover, it is still very difficult to precisely control the grasping forces on the fingertips in real scenarios due to the uncertainty from finger dynamics and object geometry [16], [17], even with the recent advanced tactile sensors such as BioTac [18].

Instead of regulating the contact forces explicitly, one may use impedance-based controllers [19]. Wimbock et al. [20] and Tahara et al. [21] developed impedance controller at the object level that prove to be very robust. Both methods rely on the concept of Virtual Frame that builds an estimate of the position and orientation of the object solely based on the positions of fingers in contact with the object. Since the virtual frame does not require explicit information on the object's geometry, it can tackle uncertainty in these estimates.

In these works, two control parameters are used for stable grasping: the stiffness $K$ and the rest length $L$. The stiffness is computed at the object's level and the rest length is the desired distance between each fingertip and the origin of the virtual frame. By varying the stiffness, the grasping forces at 
each contact point can be regulated, while the grasp configuration can be locally adjusted by changing the rest length. However, these methods rely on a heuristic to choose these two parameters for different objects. Moreover, when the object's physical properties differ from those hypothesized to generate the stable grasp, there is no strategy to guide the controller to adapt these two parameters.

In this paper, we extend the virtual frame approach of [22] and propose an adaptation scheme for the object-level impedance controller using tactile feedback $S$ to change the control parameters $K$ and $L$ so as to maintain a stable grasp. Since the objective of grasp adaptation is to ensure the stability of the final grasps, a grasp stability estimator will be designed to dynamically predict the stability of the current grasp. Compared with previous works on grasp stability estimation [5], [23], our grasp stability estimator is defined in the object frame and thus it is independent of the hand kinematics. The general framework for grasp adaptation is shown in Fig. 1. The two main components of this framework, i.e., stability estimation and grasp adaptation, are highlighted and will be detailed in the following sections.

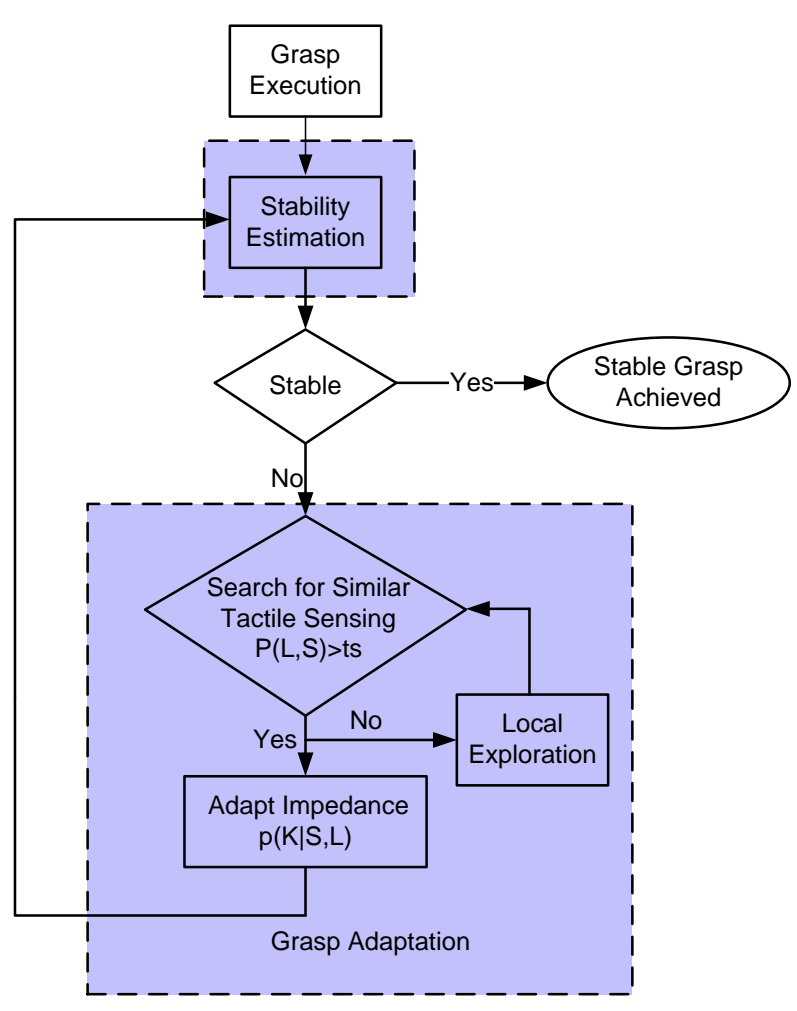

Fig. 1. The pipeline for grasp adaptation using tactile sensing, including two main components: Grasp Stability Estimation and Grasp Adaptation.

\section{Grasp Stability Estimation}

In this section, we describe our approach to learn the grasp stability estimator. This approach is similar to the previous methods by Bekiroglu et al. [23] and Dang et al. [5], but we use a different grasp feature that is directly extracted from the object-level impedance controller [22].

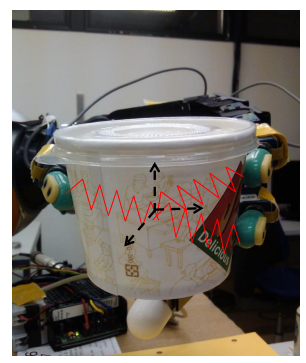

(a)

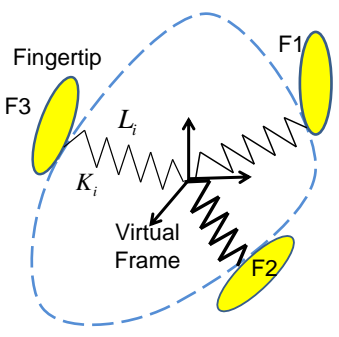

(b)
Fig. 2. The scheme for object-level impedance controller. The Virtual Frame (VF) is defined as the center of the three contact points and the controller will control the position and orientation of the VF instead of the real object configuration.

First, we describe the data used for training the grasp stability estimator. In this paper, we only consider precision grasps by three fingers, however our method can be easily extended to grasps using more than three fingers. The notation used is as follows:

- $D=\left\{\left(K^{i}, L^{i}, S^{i}\right)\right\}_{i=1 \ldots N}$ denotes a dataset with $N$ observations.

- $K^{i}=\left(K_{1}^{i}, K_{2}^{i}, K_{3}^{i}\right) \in \mathbb{R}^{3}$ denotes the grasp stiffness at each fingertip, as shown in Fig. 2.

- $L^{i}=\left(L_{1}^{i}, L_{2}^{i}, L_{3}^{i}\right) \in \mathbb{R}^{3}$ denotes the rest length at three fingertips, where $L_{j}^{i}$ is the distance from the $j$ th fingertip to the center of virtual frame.

- $S^{i}=\left(S_{1}^{i}, S_{2}^{i}, S_{3}^{i}\right) \in \mathbb{R}^{57}$ denotes the tactile reading at three fingertips, where $S_{j}^{i} \in \mathbb{R}^{19}$ is the tactile reading from the $j$-th fingertip.

The recorded data thus consist of tactile readings $S$ and parameters of the object-level controller, grasp stiffness $K$ and rest length $L$. The tactile readings are high dimensional and redundant. Thus we use the Principal Component Analysis (PCA) to reduce the dimensionality. Hereafter, we will use $S$ to denote the tactile reading after dimensionality reduction. Before the training procedure, all the data are normalized to zero mean with range $[-1,1]$.

With the recorded data, we formulate the grasp stability estimation as a one-class classification problem. This means that only the positive data, i.e, data from stable grasps, are used to learn the boundary of the stable grasp region. This is also different from previous approaches [5], [23], where both positive and negative data are used to train the model. In general, the unstable grasps in our experiments can be seen as the noisy versions of the stable grasps, i.e, too many ways to perform a bad grasp, because they do not have inherit structures to learn. Due to this, binary classification experiments did not improve the classification accuracy. Therefore it is sufficient to use only one-class approach for our data. Moreover, we noticed that in practice, it may require a huge amount of time and expense (damaging the object or the finger) to collect sufficient negative data, which would make the approach infeasible in practice. Therefore, 
in this work, we only use the positive data to learn the region of stable grasps.

In order to learn the boundary of the stable grasp region, two types of nonlinear classifiers, namely Gaussian Mixture Model (GMM) and Support Vector Machine (SVM) are used. GMM is a generative approach that models the probability distribution over data. The likelihood of a grasp $X_{*}=$ $\left(K_{*}, L_{*}, S_{*}\right)$ under a GMM model denote by $\Omega$ with $m$ Gaussian components is given by:

$$
p\left(X_{*} \mid \Omega\right)=\sum_{i=1}^{m} \pi_{i} \mathcal{N}\left(X_{*} \mid \mu_{i}, \Sigma_{i}\right)
$$

where $\pi_{i}$ is the prior of the $i$ th Gaussian component and $\mathcal{N}\left(\mu_{i}, \Sigma_{i}\right)$ is the Gaussian distribution with mean $\mu_{i}$ and covariance $\Sigma_{i}$ as follows:

$$
\mu_{i}=\left[\begin{array}{l}
\mu_{K, i} \\
\mu_{L, i} \\
\mu_{S, i}
\end{array}\right], \Sigma_{i}=\left[\begin{array}{ccc}
\Sigma_{K K, i} & \Sigma_{K L, i} & \Sigma_{K S, i} \\
\Sigma_{L K, i} & \Sigma_{L L, i} & \Sigma_{L S, i} \\
\Sigma_{S K, i} & \Sigma_{S L, i} & \Sigma_{S S, i}
\end{array}\right]
$$

A new grasp is said to be stable if its likelihood of being generated by the model is greater than a fixed threshold, i.e. $p\left(X_{*}\right)>t e$. The discrimination threshold te $\in[a, b]$ is chosen according to the ROC (Receiver Operating Characteristic) curve, where the bounds $a$ and $b$ correspond to the minimal and maximal likelihood of each Gaussian component at two standard deviation, respectively, that is,

$$
\begin{aligned}
& L_{i k_{2 \sigma}}(i)=(2 \pi)^{-\frac{d}{2}}\left|\Sigma_{i}\right|^{-\frac{1}{2}} e^{-2} \\
& a=\min _{i=1 \ldots m} \operatorname{Lik}_{2 \sigma}(i) \\
& b=\max _{i=1 \ldots m} \operatorname{Lik}_{2 \sigma}(i)
\end{aligned}
$$

As a comparison, we also applied the SVM classification to our problem. SVM for one class classification is a maximum margin classifier that attempts to separate the data from the origin with maximum margin in the feature space [24]. Here, we use SVM implementation from [25].

It is worth mentioning that other one-class classification methods [26], can also be adopted here for the grasp stability estimation. In our work, GMM is chosen because (1) it has already shown its ability at estimating region of stable grasps in the high-dimensional joint space for different hands [27] [28], (2) the grasp adaptation strategy can be naturally derived from the learned model, as will be explained in the next section.

\section{GRASP ADAPTATION}

The grasp adaptation procedure is required when the current grasp is predicted to be unstable. In this case, corrective actions should be launched and driven by the current tactile information. In our controller, grasp adaptation consists of changing the object level impedance controllers according to the tactile feedback. Specifically, we will adapt the grasp stiffness $K$ and the rest length $L$. The goal of grasp adaptation is to find a similar stable grasp using our model constructed in Section II. The following parts of this section will present two corrective actions according to the similarities between the acquired tactile readings and the training dataset.

\section{A. Impedance Adaptation}

As illustrated in Fig. 1, when a grasp $X=(K, L, S)$ is predicted to be unstable, our first adaptation strategy is to adapt the grasp stiffness. This regulates indirectly the contact forces at each finger. This adaptation strategy corresponds to a typical response in humans whereby the grip force is increased to maintain the stability of grasped (or manipulated) object [29]. Once an unstable grasp is detected, such as slippage occurring on one fingertip, the desired grasp stiffness $\hat{K}$ is predicted from the GMM through regression, given the current rest length $L$ and tactile reading $S$.

During the execution of grasp adaptation, however, we first need to check whether the current query point $q=\left[L^{T}, S^{T}\right]^{T}$ is likely enough with respect to learned model. In other words, we need to check if the current query point $q$ is close enough to the training examples. This step is required mainly because a query point with low likelihood, i.e., far away from the training examples, may give a very poor prediction that may lead to even more unstable grasps.

In order to determine if the current query point $q$ is likely under the learned model $\Omega$, we compute the Mahalanobis distance from $q$ to the center of each Gaussian component. The distance to the $i$ th component is defined as:

$$
f_{i}(q, \Omega)=\frac{1}{2}\left(q-\mu_{q, i}\right)^{T} \Sigma_{q, i}^{-1}\left(q-\mu_{q, i}\right)
$$

where $i=1, \ldots, m$ is the index of Gaussian components, $\mu_{q, i}$ and $\Sigma_{q, i}$ are the corresponding components in (1) as follows:

$$
\mu_{q, i}=\left[\begin{array}{l}
\mu_{L, i} \\
\mu_{S, i}
\end{array}\right], \Sigma_{q, i}=\left[\begin{array}{cc}
\Sigma_{L L, i} & \Sigma_{L S, i} \\
\Sigma_{S L, i} & \Sigma_{S S, i}
\end{array}\right]
$$

Note that compared to the marginal likelihood $p(q \mid \Omega)$, the Mahalanobis distance $f_{i}(q, \Omega)$ has the advantage to give the same importance to each Gaussian component, due to the absence of the prior in (4) and the fact that we normalize the distance to the center of the Gaussian. This has also the merit to avoid biasing the selection of grasps toward large Gaussian component, which may happen because of the nonuniform distribution of the training dataset.

In this work, we consider a query point $q$ is likely enough to belong to the learned model, if its Mahalanobis distance to at least one of the Gaussians of the model is below two, i.e., $\exists i, i=1, \ldots, m, f_{i}(q, \Omega)<2$. In other words, if the query point is inside the two standard deviations of any Gaussians of the model, then it is considered to be close enough to the learned model.

When the current query point $q$ is likely enough under the model, the expected grasp stiffness is obtained by taking the expectation over the conditional distribution, $p(\hat{K} \mid L, S, \Omega)$ [30], which can be computed as follows:

$$
E\{p(\hat{K} \mid L, S, \Omega)\}=\sum_{i=1}^{m} h_{i}\left(\mu_{k, i}+\Sigma_{K q, i} \Sigma_{q, i}^{-1}\left(q-\mu_{q, i}\right)\right)
$$

where $\Sigma_{K q, i}=\left[\begin{array}{c}\Sigma_{K L, i} \\ \Sigma_{K S, i}\end{array}\right]$, and $h_{i}=\frac{\pi_{i} \mathcal{N}\left(q \mid \mu_{q, i}, \Sigma_{q, i}\right)}{\sum_{j=1}^{m} \pi_{j} \mathcal{N}\left(q \mid \mu_{q, j}, \Sigma_{q, j}\right)}$.

The expected grasp stiffness value will be thus set directly to the object level impedance controller, as shown in Fig. 1. 


\section{B. Local Exploration}

When the current query point $q$ is far away from all the training examples, a second adaptation strategy takes place to project the current query point to the closest point $q_{*}$ in the training dataset. Since in the controller only the rest length is required to determine the grasp configuration, we move the current grasp configuration towards the closest center of the Gaussian components, which is chosen according to the same distance metric defined above (4). The rest length of the closest center of Gaussian component is denoted as $L^{c}=\left[L_{1}^{c}, L_{2}^{c}, L_{3}^{c}\right]^{T}$.

However, differently from the grasp stiffness, we can not simply change these parameters in the controller since the definition of the virtual frame depends on the contact position at each fingertip. Moreover, the kinematics of each finger and the local geometry of the object surface will also impose several constraints on the possible movements of each fingertip. We therefore adopt an iterative approach by defining the following objective function:

$$
J=\sum_{i=1}^{3}\left(L_{i}-L_{i}^{c}\right)^{2}
$$

where $L=\left[L_{1}, L_{2}, L_{3}\right]^{T}$ is the current rest length. In this work, we only consider the adaptation motion from one of the fingers, denoted here as finger 1, see Fig. 3. For a more general case with multi-fingered grasp (more than three fingers), a more elaborate strategy that considers each finger's workspace and the grasp stability would be required to determine which finger should be used for adaptation.

In order to minimize the objective function, the gradient of the objective function with respect to the position of finger 1 , i.e., $P_{1}=\left[X_{1}, Y_{1}, Z_{1}\right]$, is given as $v=\left[\frac{\partial J}{\partial X_{1}}, \frac{\partial J}{\partial Y_{1}}, \frac{\partial J}{\partial Z_{1}}\right]^{T}$, where each component of $v$ is computed as:

$$
\frac{\partial J}{\partial X_{1}}=\sum_{i=1}^{3} \frac{\partial J}{\partial L_{i}} \frac{\partial L_{i}}{\partial X_{1}}
$$

$\frac{\partial J}{\partial L_{i}}$ and $\frac{\partial L_{i}}{\partial X_{1}}$ can be easily computed from the definition of $J$ in (7) and the definition of $L_{i}$ as given in [22].

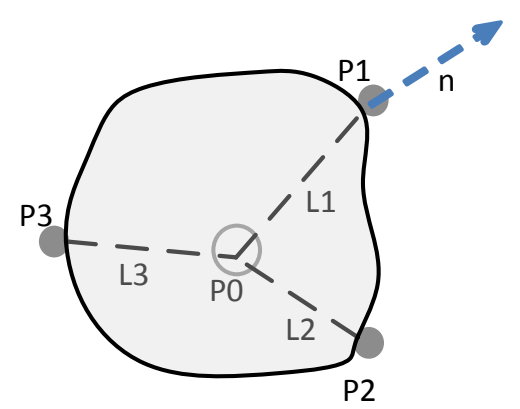

Fig. 3. The scheme for local object exploration. Only finger 1 is used here for local exploration and the normal direction obtained from tactile sensing is also used for guiding the exploration directions.

Due to the constraint of the object surface, the fingertip 1 can not penetrate inside the object surface. Therefore we need to project the gradient onto the tangential surface at fingertip 1: $v^{*}=v-\langle v, n\rangle n$, where $n$ is the normal direction at fingertip 1 . Then the next desired position of finger 1 is given by: $P_{1}^{*}=P_{1}-\alpha v^{*}$, where $\alpha \in(0,1)$ is the step size and is manually set as 0.03 in our experiment ${ }^{1}$. In order to move finger 1 to the desired position, we implement a fingertip impedance controller for finger 1 , which is superimposed on the object-level impedance controller.

It is worth noticing that during the local exploration, only the rest length $L$ can be controlled. This does not guarantee that a similar query point $q$, consisting of the rest length $L$ and the tactile sensing $S$, can be always found. To this end, a maximum number of steps for local exploration is set in our controller, i.e., $N_{\max }=5$. If a similar query point cannot be found within $N_{\max }$ times steps, our controller will recompute the closest center of the Gaussian components according to (4) and repeat the local exploration loop.

\section{EXPERIMENTAL EVALUATION}

In this section, we demonstrate the effectiveness of our approach on several everyday objects. We first present the experimental setup and the data collection procedure, then present the results on grasp stability estimation and grasp adaptation.

\section{A. Setup and Data Collection}

As shown in Fig. 4, we use a 16 DOFs (degree of freedom) Allegro Hand from Simlab ${ }^{2}$ with four fingers. Each finger has four independent torque-controlled joints. In our experiments we only use three of these four fingers. Each fingertip has been equipped with the tactile sensor BioTac from SynTouch ${ }^{3}$. BioTac can provide multi-modal tactile information, such as vibration, temperature and pressure. Here, we only use the 19 dimensional pressure data from the electrode impedances, which are related to the contact features such as contact force, contact location and deformation. The sampling rate is $100 \mathrm{~Hz}$ in our experiments.

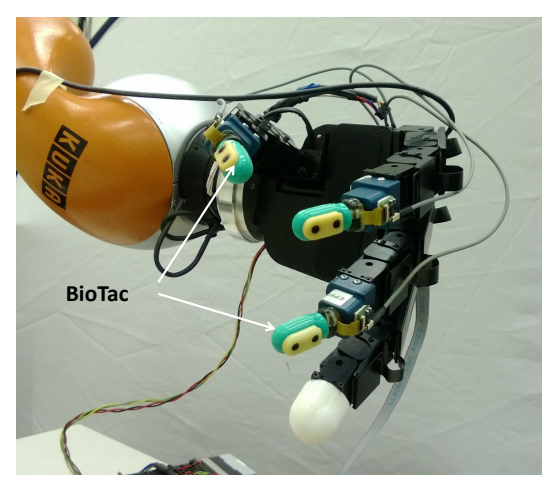

Fig. 4. Allegro Hand equipped with BioTac on the fingertips.

\footnotetext{
${ }^{1}$ Note that there is a trade-off here. If $\alpha$ is small, the object may fall before finding a stable grasp. If $\alpha$ is too large, the finger may overshoot the desired position or move out of the object surface.

${ }^{2} \mathrm{http}: / /$ www.simlab.co.kr/Allegro-Hand.htm

${ }^{3}$ http://www.syntouchllc.com/
} 


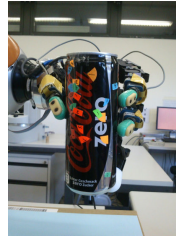

(a) cola can

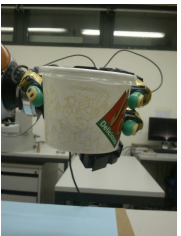

(b) food box

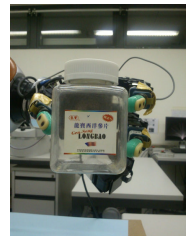

(c) box

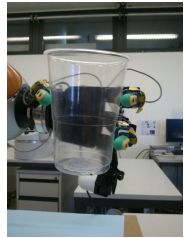

(d) cup
Fig. 5. The four objects used in the experiments for collecting training dataset.

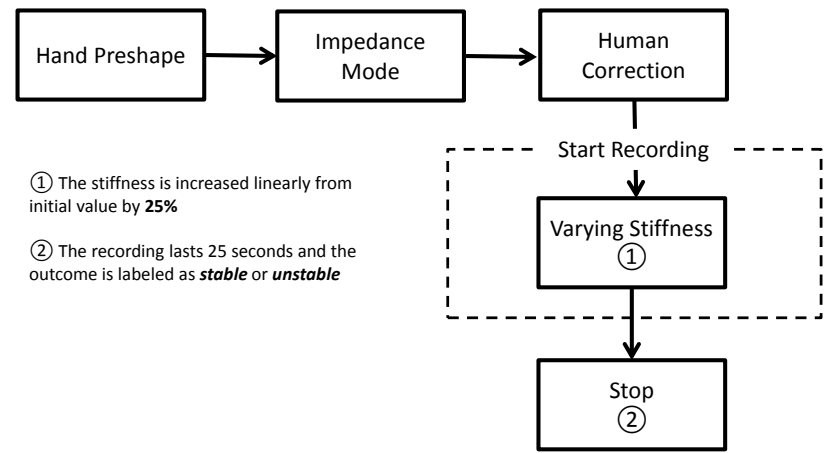

Fig. 6. The data collection procedure. Only one hand preshape is used in our experiment, as shown in Fig. 4. The object is put inside the hand and the control mode is changed to impedance mode. One or more fingertips' position is locally adjusted to set an initial stable grasp. This step is also required since we want to vary the rest length for each trial.

Our primary goal in this paper is to deal with physical uncertainties and therefore we assume that the object is already grasped with a given preshape, Fig. 4 . The geometric information about the object is not known a-priori. Four different objects are used in our experiments, shown in Fig. 5. For each object we have five different weights by filling them with different amount of pepper, see Table I. The initial grasp stiffness is manually chosen as a minimal value that can grasp the object in a stable way. Before recording, the initial grasp may be slightly adjusted by the human to change the grasp configuration, i.e., to change the possible value of the rest lengths. Once recording has started, the stiffness is increased linearly from the initial value to $125 \%$ of the initial value with $10 \%$ random noise. Both the change of initial grasp configuration and the increase of grasp stiffness in the experiments are aiming at increasing the variety of our collected data.

TABLE I

THE WEIGHTS OF DIFFERENT OBJECTS DURING TRAINING

\begin{tabular}{|c|c|c|c|c|r|}
\hline object & \multicolumn{5}{|c|}{ object weight(g) } \\
\hline cola can & 16 & 38 & 50 & 66 & 80 \\
food box & 10 & 26 & 52 & 68 & 88 \\
box & 34 & 56 & 76 & 99 & 121 \\
cup & 10 & 49 & 98 & 135 & 155 \\
\hline
\end{tabular}

The recording procedure lasts 25 seconds for each trial and the whole trial is repeated 5 times for each object weight. Fig. 6 provides an outline of the data collection. In total, we collected $4 \times 5 \times 5 \times 25 \times 100=250000$ positive examples.
For each object, we also collected several negative examples by either setting the initial stiffness to a very small value or filling much more pepper into the objects. In total, we have 37500 negative examples.

\section{B. Results for Grasp Stability Estimation}

The dataset is divided into training and test sets. For the first three objects (cola can, food box, box), one weight is selected randomly and the data collected with this weight is used as the test set. For the cup, all the collected data are grouped into test set. All the negative datapoints are also used for testing. The tactile data has originally $19 \times$ $3=57$ dimensions and the dimensionality is reduced to 8 dimensions based on PCA, hence in total the data has $8+3+3=14$ dimensions

For training the model defined by (1), the number of Gaussian components, $m$, is selected using Bayesian Information Criteria (BIC) and set to 16 in our experiments, $m=16$. The other parameters in GMM are learned using the EM algorithm. Figure 7 shows the learned stable grasp region projected onto the first two principal components of the tactile reading. Examples of stable grasps and unstable grasps are given for each object. As shown in Fig. 8, in these projected lower dimensions, some unstable grasps can be easily separated, such as unstable grasp 2, (grasp of food box). This grasp is unstable because one finger (finger 2) loses contact with the object, see the right upper picture in Fig. 7. But in most other cases, when the object starts to slip or tilt slowly from the hand, these unstable grasps cannot be easily separated from stable grasps in the projected lower dimensions. We need to use all the dimensions to compute the marginal likelihood according to (1) and compare it with the threshold $t e$.

In order to select the threshold te in (1), we vary the value of $t e$ in (3) with $a=-708.4$ and $b=-35.30$ (The logarithmic likelihood is used here). The ROC curve for the test dataset is shown in Fig. 9. The variance on the curve is calculated based on 5 fold cross-validation.

For our application, false positives should be avoided at all costs, as they correspond to the cases where an unstable grasp is mistaken for a stable grasp. For this reason, we chose a very high te value which corresponds to $T P R=82.27 \%$ and $F P R=15.01 \%$. This result is comparable with the results obtained in [23] for unknown objects.

For comparison, the SVM for one-class classification is also used to predict the grasp stability. We use the RBF (radial basis function) kernel and the parameters are selected using multi-scale grid search. The best performance SVM can obtain is: $T P R=84.66 \%$ and $F P R=29.72 \%$. Although the true positive rates are similar, the SVM has a higher false positive rate. This can be explained by the fact that one-class SVM only tries to separate the training data with origin in the feature space [26], which is a considerable loose constraint compared with a GMM that attempts to model the density of the stable grasp region. 


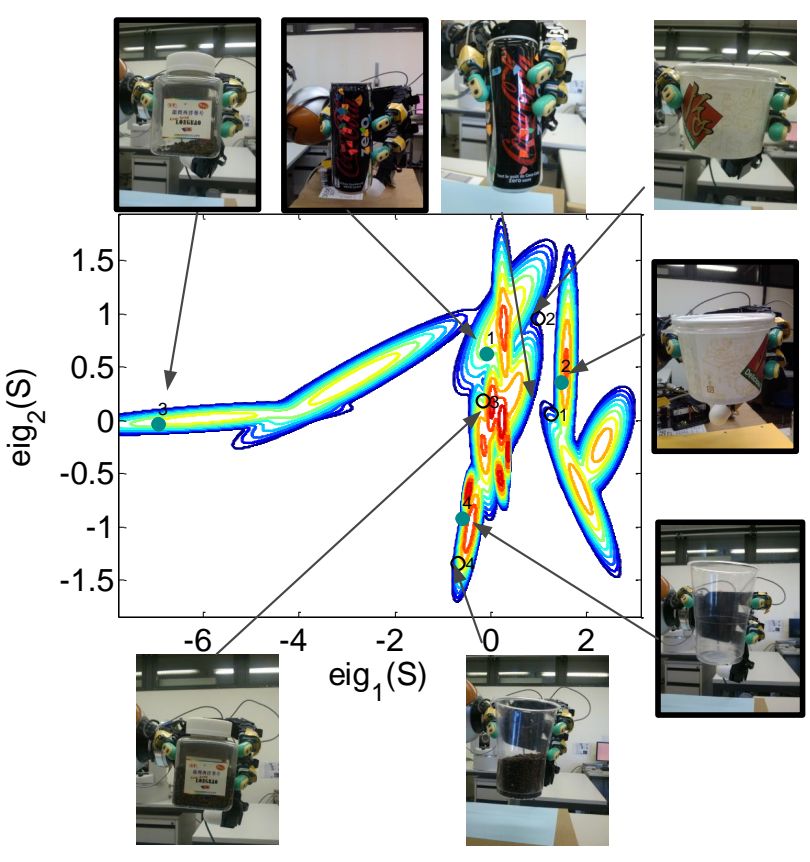

Fig. 7. The density distribution of the learned GMM model. The axes correspond to the projection of the tactile sensing $S$ on the first two principal components. Contours correspond to the isocurve with constant marginal likelihood value $p(S \mid \Omega)$. Solid dots denote the stable grasps and circles denote the unstable grasps.

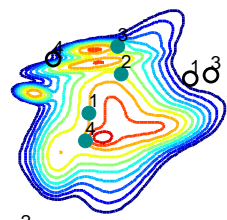

子

(a) $\operatorname{eig}_{7}(S)$ vs $L_{1}$

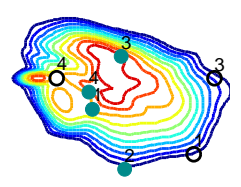

子

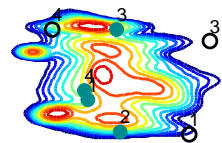

$0^{2}$
Fig. 8. The density distribution of the learned GMM model projected into lower dimensions. The $\mathrm{X}$-axis corresponds to the 7 th dimension of the tactile readings and the Y-axis corresponds to the dimension of $L_{1}, L_{2}$ and $L_{3}$, respectively. Contours correspond to the isocurve with constant marginal likelihood value $p(S \mid \Omega)$. Solid dots denote the stable grasps and circles denote the unstable grasps.

\section{Results for Grasp Adaptation}

To test the validity of our grasp adaptation strategy, first we only use the collected negative datapoints to demonstrate that these grasps can be predicted to be unstable grasps. Also, depending on their similarities with the training dataset, different adaptation strategies should be able to react. For all the 37500 negative datapoints, only $8.70 \%$ of them are misclassified as stable grasps. $33.70 \%$ of them require the impedance adaptation while $57.60 \%$ of them require the local exploration.

We also tested our grasp adaptation approach on the real robotic hand with different objects, see Fig. 10-15. When the object weights are changing by adding pepper or disturbed by

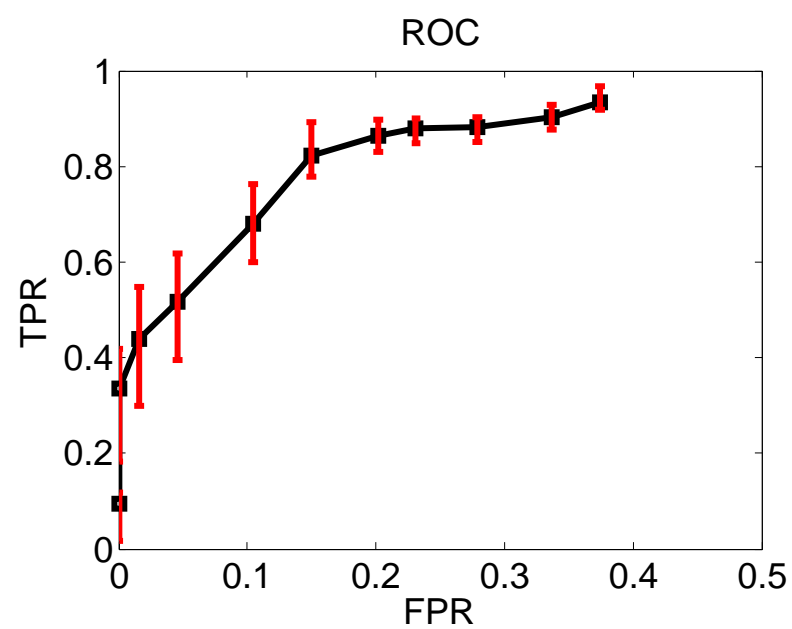

Fig. 9. ROC curve to select the threshold te.

a human ${ }^{4}$, the grasp adaptation strategy is triggered to keep the grasp stable, either by varying the grasp stiffness or by changing the location of finger 1 . Taking the test of the cup as an example, see Fig. 11, with the increase of the weight, first the stiffness is increasing until the distance computed from (4) is larger than 2. At the second stage (shown as red dots), the finger 1 starts to explore local area until the query point is close again. Finally, the stiffness is changed again to find a stable grasp.

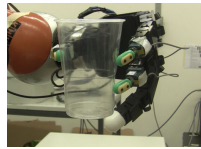

(a)

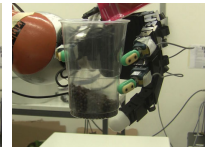

(b)

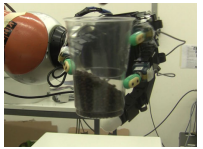

(c)

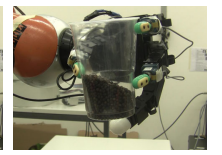

(d)
Fig. 10. A snapshot for the experiment on the cup. (a) The initial grasp stiffness is set manually. (b) Pepper was poured into the cup to change the cup's weight. (c) Finger 1 is changing its location for local exploration. (d) The new grasp configuration after the exploration of finger 1 .

To quantify the results of our grasp adaptation strategy, we compare the maximal object weights that the grasp can support with and without the grasp adaptation strategy, see Table II. We use the same initial grasp configuration and grasp stiffness for each object as in the data collection procedure, i.e., the setup for data collection in the first column of Table I. The object weight is still varying by adding pepper and the object is considered as unstable once there is a steady noticeable slippage between the fingertips and the object. Each object is tested five times, both with grasp adaptation and without grasp adaptation.

In Table II, each row corresponds to the average value of the maximal object weight (grams) that the grasp can support for each object, with (with) and without (w/o) grasp adaptation. The standard deviation during the five experiments is also computed and given as mean \pm std. The comparison of the results shows that the grasp adaptation

\footnotetext{
${ }^{4}$ We use human perturbation to simulate the change of the object weight.
} 


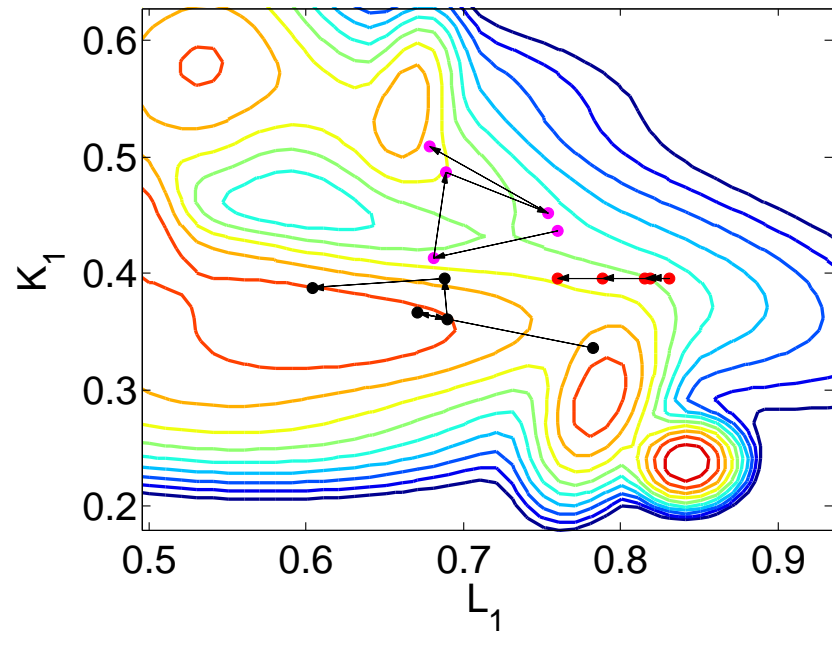

Fig. 11. The joint density distribution of the dimensions $L_{1}$ and $K_{1}$; The black dots correspond to the impedance adaptation stage for the testing of the cup ( Fig. 10(b)). Red dots correspond to the local exploration stage and magenta dots correspond to the impedance adaptation after local exploration. For each stage, we only plot 5 datapoints.

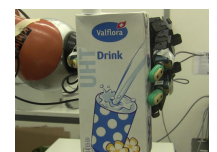

(a)

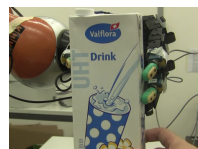

(b)

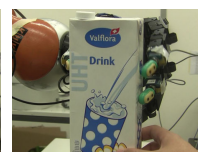

(c)

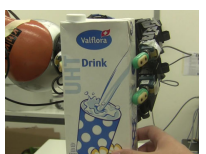

(d)
Fig. 12. A snapshot for the experiment on the milkbox. A human is pulling the milkbox downwards (b) and at the time shown in (c), finger 1 starts to change its location in order to keep the stability of the grasp.

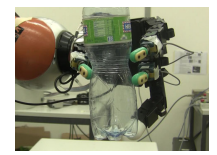

(a)

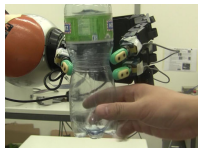

(b)

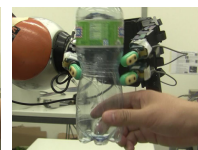

(c)

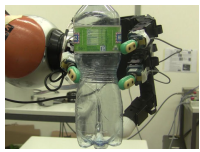

(d)
Fig. 13. A snapshot for the experiment on the water bottle.

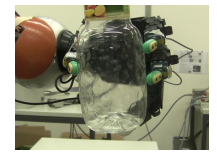

(a)

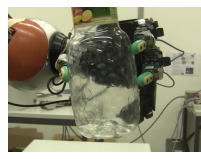

(b)

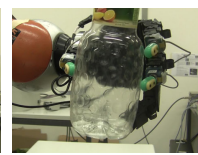

(c)

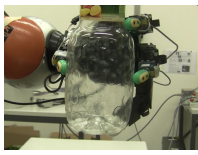

(d)
Fig. 14. A snapshot for the experiment on the juice bottle. A human is trying to push the bottle downwards from the top of the bottle (b) and finger 1 starts to adapt its location at the moment shown in (c).

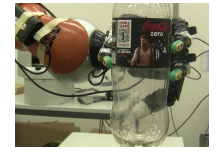

(a)

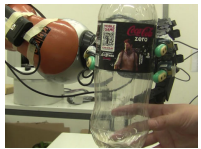

(b)

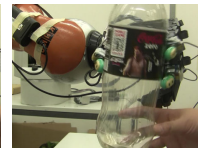

(c)

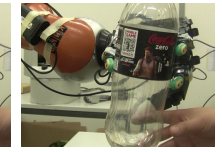

(d)
Fig. 15. A snapshot for the experiment on the cola bottle.

can have a significant improvement on the maximal weight that a grasp can support.
TABLE II

THE COMPARISON OF THE SUPPORTED OBJECT WEIGHTS (WITH VS. W/O GRASP ADAPTATION)

\begin{tabular}{|c|c|c|c|c|}
\hline obj. & cola can & food box & box & cup \\
\hline w/o & $17.2 \pm 1.92$ & $12.8 \pm 0.84$ & $37.2 \pm 2.59$ & $15.0 \pm 2.55$ \\
\hline with & $69.0 \pm 6.52$ & $84.0 \pm 3.80$ & $121.2 \pm 9.20$ & $146.4 \pm 5.46$ \\
\hline
\end{tabular}

\section{Discussion}

In our controller, since the virtual frame does not assume prior information about the object's shape, this may lead to problems for local exploration. The exploring finger 1 may move out of the object surface after the local exploration, although this problem can be avoided by detecting the loss of contact and moving the finger back to the previous contact position. Also, instead of using only finger 1 , other fingers can also be used for local exploration, which will be especially useful when the object is grasped by more than 3 fingers. This requires a more complicated exploration strategy as well as a grasp planning strategy that takes each finger's adaptability into account during the planning stage [31].

Another issue is about the implementation of local exploration. At present, to do the local exploration, we only implemented a fingertip impedance controller to move finger 1 to the desired position. However, we found that in practice it is not so trivial to choose the proper parameters for the fingertip impedance controller. As shown in Fig. 10 and Fig. 14, the finger 1 may "jump" to the desired position, which sometimes leads to unstable grasps due to either the loss of contact during jump or the large impact during contact. It is more reasonable to implement a contour following controller that can move the exploring finger (finger 1) to the desired position by following the object's contour. However, since the object is grasped and supported by the other fingers during the exploration, the force that the exploring finger applies on the surface should be adapted to the stiffness of the grasped object.

\section{CONClusion}

This paper presented a new framework for grasp adaptation under physical uncertainties of the grasped objects. The adaptation strategy is derived from an object-level impedance controller and learned with training data that is generated using a real robotic hand. We first formulated the grasp stability estimation as a one-class classification problem. A Gaussian Mixture Model is used to model the region of the stable grasps. During the grasp execution, if a grasp is predicted to be unstable, two different adaptation strategies, i.e., impedance adaptation and local exploration, are selectively triggered according to the similarity between the current unstable grasp and the examples in the stable grasp regions. The effectiveness of our approach is validated on the Allegro hand equipped with BioTac tactile sensors for the experiments. 


\section{ACKNOWLEDGMENT}

This work was supported by the European Union Seventh Framework Programme FP7/2007-2013 under grant agreement $\mathrm{n}^{\mathrm{o}} 288533$ ROBOHOW.COG.

\section{REFERENCES}

[1] R. C. Brost, "Planning robot grasping motions in the presence of uncertainty," Tech. Rep. CMU-RI-TR-85-12, Robotics Institute, Pittsburgh, PA, July 1985.

[2] Y. Zheng and W.-H. Qian, "Coping with the grasping uncertainties in force-closure analysis.," International Joournal of Robotic Research, vol. 24, no. 4, pp. 311-327, 2005.

[3] B. Kehoe, D. Berenson, and K. Goldberg, "Toward cloud-based grasping with uncertainty in shape: Estimating lower bounds on achieving force closure with zero-slip push grasps," in Proceedings of International Conference on Robotics and Automation (ICRA), 2012.

[4] V. N. Christopoulos and P. R. Schrater, "Handling shape and contact location uncertainty in grasping two-dimensional planar objects," in Proceedings of International Conference on Intelligent Robots and Systems (IROS), 2007.

[5] H. Dang and P. Allen, "Stable grasping under pose uncertainty using tactile feedback," Autonomous Robots, pp. 1-22, 2013.

[6] J. Laaksonen, E. Nikandrova, and V. Kyrki, "Probabilistic sensor-based grasping," in Proceedings of International Conference on Intelligent Robots and Systems (IROS), 2012.

[7] K. Hsiao, S. Chitta, M. Ciocarlie, and E. Jones, "Contact-reactive grasping of objects with partial shape information," in Proceedings of International Conference on Intelligent Robots and Systems (IROS), 2010.

[8] J. Felip and A. Morales, "Robust sensor-based grasp primitive for a three-finger robot hand," in Proceedings of International Conference on Intelligent Robots and Systems (IROS), 2009.

[9] J. Kim, K. Iwamoto, J. Kuffner, Y. Ota, and N. Pollard, "Physically based grasp quality evaluation under pose uncertainty," IEEE Transactions on Robotics, vol. 29, no. 6, pp. 1424-1439, 2013.

[10] H. Dang and P. Allen, "Grasp adjustment on novel objects using tactile experience from similar local geometry," in Proceedings of International Conference on Intelligent Robots and Systems (IROS), 2013.

[11] J. Steffen, R. Haschke, and H. Ritter, "Experience-based and tactiledriven dynamic grasp control," in Proceedings of International Conference on Intelligent Robots and Systems (IROS), 2007.

[12] S. El-Khoury, M. Li, and A. Billard, "Bridging the gap: One shot grasp synthesis approach," in Proceedings of International Conference on Intelligent Robots and Systems (IROS), 2012.

[13] S. El-Khoury, M. Li, and A. Billard, "On the generation of a variety of grasps," Robotics and Autonomous Systems, vol. 61, no. 12, pp. 13351349, 2013.

[14] J. M. Romano, K. Hsiao, G. Niemeyer, S. Chitta, and K. J. Kuchenbecker, "Human-inspired robotic grasp control with tactile sensing.," IEEE Transactions on Robotics, vol. 27, no. 6, pp. 1067-1079, 2011.

[15] T. Schlegl, F. Freyberger, S. Haidacher, F. Pfeiffer, M. Buss, and G. Schmidt, "Compensation of discrete contact state errors in regrasping experiments with the tum-hand," in Proceedings of International Conference on Intelligent Robots and Systems (IROS), 1999.
[16] K.-C. Nguyen and V. Perdereau, "Fingertip force control based on max torque adjustment for dexterous manipulation of an anthropomorphic hand," in Proceedings of International Conference on Intelligent Robots and Systems (IROS), 2013.

[17] W. Yang, M. Li, and X. Zhang, "Robust robotic grasping force optimization with uncertainty.," in ICIRA, vol. 6425 of Lecture Notes in Computer Science, pp. 264-275, Springer, 2010.

[18] N. Wettels, V. J. Santos, R. S. Johansson, and G. E. Loeb, "Biomimetic tactile sensor array.," Advanced Robotics, vol. 22, no. 8, pp. 829-849, 2008.

[19] T. Yoshikawa, "Multifingered robot hands: Control for grasping and manipulation," Annual Reviews in Control, vol. 34, no. 2, pp. 199 208, 2010.

[20] T. Wimbock, C. Ott, and G. Hirzinger, "Analysis and experimental evaluation of the intrinsically passive controller (IPC) for multifingered hands," in Proceedings of International Conference on Robotics and Automation (ICRA), 2008.

[21] K. Tahara, S. Arimoto, and M. Yoshida, "Dynamic object manipulation using a virtual frame by a triple soft-fingered robotic hand," in Proceedings of International Conference on Robotics and Automation (ICRA), 2010.

[22] M. Li, H. Yin, K. Tahara, and A. Billard, "Learning object-level impedance control for robust grasping and dexterous manipulation," in Proceedings of International Conference on Robotics and Automation (ICRA), 2014.

[23] Y. Bekiroglu, J. Laaksonen, J. A. Jorgensen, V. Kyrki, and D. Kragic, "Assessing grasp stability based on learning and haptic data," IEEE Transactions on Robotics, vol. 27, no. 3, pp. 616-629, 2011.

[24] B. Schölkopf, J. C. Platt, J. C. Shawe-Taylor, A. J. Smola, and R. C. Williamson, "Estimating the support of a high-dimensional distribution," Neural Comput., vol. 13, pp. 1443-1471, July 2001.

[25] C.-C. Chang and C.-J. Lin, "LIBSVM: A library for support vector machines," ACM Transactions on Intelligent Systems and Technology, vol. 2, pp. 27:1-27:27, 2011. Software available at http://www.csie.ntu.edu.tw/ cjlin/libsvm.

[26] V. Chandola, A. Banerjee, and V. Kumar, "Anomaly detection: A survey," ACM Comput. Surv., vol. 41, pp. 15:1-15:58, July 2009.

[27] B. Huang, S. El-Khoury, M. Li, J. J. Bryson, and A. Billard, "Learning a real time grasping strategy.," in Proceedings of International Conference on Robotics and Automation (ICRA), 2013.

[28] E. L. Sauser, B. Argall, G. Metta, and A. Billard, "Iterative learning of grasp adaptation through human corrections.," Robotics and Autonomous Systems, vol. 60, no. 1, pp. 55-71, 2012.

[29] R. S. Johansson, "Sensory input and control of grip," in Novartis Foundation 218: Sensory Guidance of Movement, pp. 45-63, 1998.

[30] D. A. Cohn, Z. Ghahramani, and M. I. Jordan, "Active learning with statistical models.," Journal of Artificial Intelligence Research, vol. 4, pp. 129-145, 1996.

[31] K. Hang, J. A. Stork, and D. Kragic, "Hierarchical fingertip space for multi-fingered precision grasping," in Proceedings of International Conference on Intelligent Robots and Systems (IROS), 2014. 